BMJ Open Sport \& Exercise Medicine

\title{
Evaluating the effects of arthroscopic Bankart repair and open Latarjet shoulder stabilisation procedures on shoulder joint neuromechanics and function: a single-centre, parallel-arm trial protocol
}

\author{
Aaron Fox (10 ,1,2 Jason Bonacci, ${ }^{1}$ Stephen D Gill, ${ }^{2,3}$ Richard S Page ${ }^{2,3,4}$
}

\begin{abstract}
To cite: Fox A, Bonacci J, Gill SD, et al. Evaluating the effects of arthroscopic Bankart repair and open Latarjet shoulder stabilisation procedures on shoulder joint neuromechanics and function a single-centre, parallel-arm trial protocol. BMJ Open Sport \& Exercise Medicine 2021;7:e000956. doi:10.1136/ bmjsem-2020-000956
\end{abstract}

Accepted 18 January 2021

\section{Check for updates}

C) Author(s) (or their employer(s)) 2021. Re-use permitted under CC BY-NC. No commercial re-use. See rights and permissions. Published by BMJ.

${ }^{1}$ Centre for Sport Research, School of Exercise and Nutrition Sciences, Deakin University, Waurn Ponds, Victoria, Australia ${ }^{2}$ Barwon Centre for Orthopaedic Research and Education (BCORE), Barwon Health, Geelong, Victoria, Australia

${ }^{3}$ School of Medicine, Deakin University, Geelong, Victoria, Australia

${ }^{4}$ Orthopaedic Department, University Hospital Geelong, Geelong, Victoria, Australia

Correspondence to Dr Aaron Fox; aaron.f@deakin.edu.au

\section{ABSTRACT}

Introduction Shoulder instability injuries are common in sports involving collisions and overhead movements. Arthroscopic Bankart repair and the open Latarjet are two commonly used surgical stabilisation procedures. There is a lack of knowledge surrounding movement strategies, joint loading and muscle strength after each of these procedures. This study will compare: (1) shoulder joint neuromechanics during activities of daily living and an overhead sporting task; (2) shoulder range of motion; (3) shoulder strength; and (4) self-reported shoulder function and health status, between individuals who have undergone an arthroscopic Bankart repair versus open Latarjet.

Methods and analysis This is a prospective cohort, single-centre, non-randomised parallel arm study of surgical interventions for athletic shoulder instability injuries. Thirty participants will be recruited. Of these, 20 will have experienced one or more traumatic shoulder instability injuries requiring surgical stabilisation-and will undergo an arthroscopic Bankart repair or open Latarjet procedure. The remaining 10 participants will have no history of shoulder instability injury and act as controls. Participants will undergo baseline testing and be followed up at 3, 6 and 12 months. A two-way (groupxtime) analysis of variance with repeated measures on one factor (ie, time) will compare each outcome measure between groups across time points.

Ethics and dissemination This study was approved by the Barwon Health and Deakin University Human Research Ethics Committees. Outcomes will be disseminated through publications in peer-reviewed journals and presentations at relevant scientific conferences.

Trial registration number Australian and New Zealand Clinical Trials Registry (ACTRN12620000016932).

\section{INTRODUCTION}

Traumatic anterior shoulder instability is common in sports involving collisions and overhead movements, such as Australian rules football, American football and rugby union. $^{1-3}$ These injuries occur when the humeral head is forced out of the shoulder joint socket (ie, subluxation or dislocation). ${ }^{4}$ Avulsion of the labral attachment of the anteroinferior glenohumeral ligament between the 3 and 5 o'clock positions (ie, Bankart lesion) is the most commonly observed pathology in traumatic anterior shoulder instability. ${ }^{5}$ Glenoid bone loss or defects, along with humeral bone defects (ie, Hill-Sachs lesions) can also occur. ${ }^{6-8}$ The incidence of shoulder instability is increasing, ${ }^{9}$ recurrent injuries are common, ${ }^{10}$ and patients can experience arm pain and weakness with a shoulder that easily dislocates with minimal movement or force. ${ }^{4}$ These symptoms can lead to repeated hospital admissions, decreased ability to participate in activity or sport, fewer employment opportunities and a reduction in overall health. ${ }^{11}$

Effective clinical care and rehabilitation is vital to avoid recurrent injuries, reduced shoulder function and joint degradation later in life. ${ }^{4}$ Surgery is commonly used to address intra-articular pathology, restore shoulder function and correct stability following shoulder instability injuries. Arthroscopic procedures are typically preferred due to their minimally invasive and conservative nature. ${ }^{12}$ Arthroscopic Bankart repair (ie, anatomic repair of Bankart lesion) is commonly used ${ }^{13}$ and aims to restore the labrum and re-establish appropriate tension in the joint capsule and ligaments. ${ }^{14}$ In certain cases, open (ie, surgery via a large incision) procedures are preferred. ${ }^{14}$ The Latarjet procedure is a nonanatomical, open shoulder reconstruction surgery involving a bone block via transfer of the inferior surface of the coracoid process to the anterior glenoid with the attached 
conjoint tendon. ${ }^{15}$ The Latarjet procedure is more commonly used in cases with significant glenoid bone loss, large Hill-Sachs lesions, or combined glenoid and humeral bone defects ${ }^{14}$-but is emerging as a preferred option for operative shoulder stabilisation in contact sport settings. ${ }^{16}$

A proposed benefit of the Latarjet procedure is the reduced risk of recurrent instability, with studies reporting a reduced number or rate of recurrent subluxations and dislocations in comparison with other (eg, arthroscopic Bankart repair, up to $10 \%$ at up to 8 years ${ }^{17}$ ) reconstruction procedures. ${ }^{18}{ }^{19}$ Neyton et $a l^{20}$ found no recurrent subluxations or dislocations over a mean follow-up time of 12 years in a group of rugby union players who underwent the Latarjet procedure. In addition, while arthroscopic repair can restore shoulder stability and function, not all athletes who undergo this procedure return to the same level of sport. ${ }^{21}$ While the Latarjet procedure may reduce the risk of recurrent instability and promote return to sport, bone block procedures incur higher complication rates (ie, $7.2 \%-13.6 \%$ vs $0.5 \%-6.2 \%$ for non-bone block procedures) ${ }^{22}$ and may also contribute to postoperative osteoarthritis due to impingement on articular cartilage. ${ }^{23}$ The Latarjet procedure requires high precision and subtle variations in surgical technique (eg, coracoid bone graft placement and orientation, graft fixation, subscapularis reattachment location) may impact graft strength, the loads experienced at the shoulder and the likelihood of degenerative changes arising. ${ }^{24-26}$ These surgical choices can impact on the structure and function of the shoulder joint following reconstruction, and subsequently affect glenohumeral joint loads during movement. There is, however, an absence of studies investigating the joint loads experienced during movement following shoulder stabilisation procedures. Knowledge of how shoulder stabilisation procedures impact joint loading during movement can aid in understanding the risk and for joint instability and degenerative changes following procedures, and provide an adequate target for rehabilitation efforts. Three-dimensional analyses, combined with musculoskeletal modelling and simulation, afford the opportunity to examine muscle function, and joint loading and stability during movement.

Various differences in self-reported (ie, Rowe scores) and objective measures (ie, range of motion) of shoulder function have been reported between arthroscopic and Latarjet procedures. A systematic review and metaanalysis by An $e t a l^{27}$ found a weighted mean difference of 3.29 (95\% CI 2.37 to 4.20 ) in favour of the Latarjet procedure over Bankart repairs in self-reported Rowe scores (ie, a scoring system assessing shoulder stability, motion and function). This value does, however, fall below the recommended minimal clinically important difference (ie, 9.7) for the Rowe score. ${ }^{28}$ The review also found external rotation range of motion loss was less in Latarjet procedures compared with Bankart repairs $\left(11.9^{\circ}\right.$ vs $20.9^{\circ}$; weighted mean difference $=5.75,95 \%$ CI 4.73 to 6.77) ${ }^{27}$ However, these data encompassed studies that examined both arthroscopic and open Bankart repairs, as well as studies that examined traditional and variants (ie, Bristow-Latarjet, open Bristow) of the Latarjet procedure. To our knowledge, only one study ${ }^{19}$ has compared outcomes between arthroscopic Bankart repair and the traditional open Latarjet procedure. In this retrospective study, the recurrent instability rate was lower and Rowe scores were higher in the open Latarjet group, with no data on range of motion reported. ${ }^{19}$ There appears to be a lack of prospective evaluation comparing relevant measures of shoulder function between arthroscopic Bankart repairs and traditional open Latarjet procedures. Further, none of the aforementioned studies have examined shoulder strength - an important aspect of shoulder function and stability, which has implications for rehabilitation for glenohumeral instability. ${ }^{29}$ There is also no indication across this literature regarding how variations in measures of shoulder joint function (self-reported or objective) impact on shoulder joint neuromechanics (ie, kinematics and kinetics, joint loading, muscle activation and forces) during movement tasks.

The aim of this study is to compare outcomes between individuals who have undergone an arthroscopic Bankart repair versus a traditional open Latarjet procedure (herein referred to simply as a Latarjet procedure), while also referencing these outcomes to healthy uninjured controls. The outcomes that will be examined in this study are: (1) shoulder joint neuromechanics (ie, shoulder joint loads, kinematics, kinetics, and muscle activation and forces) during activities of daily living and an overhead sporting task; (2) shoulder range of motion; (3) shoulder strength; and (4) self-reported shoulder function and health status. A metric of glenohumeral joint stability will be derived from calculated shoulder joint loads during movement tasks and used as the primary neuromechanical outcome measure between the two procedures. Knowledge of how the two procedures impact shoulder joint neuromechanics and glenohumeral stability during relevant movement tasks involving the shoulder may provide evidence for the use of a surgical procedure in restoring movement capacity, reducing the potential for future instability and minimising joint degradation over time. Further, understanding how shoulder joint function and the relationship of these to joint loading and stability during movement varies between procedures can assist in designing more targeted and specific rehabilitation strategies following shoulder stabilisation.

\section{METHODS AND ANALYSIS Study design}

This study is a prospective, single-centre, non-randomised parallel-arm trial. The study will be conducted at a large regional health service in Victoria, Australia. The study will include baseline testing, and follow-up testing at 3, 6 and 12 months (see study design in figure 1). Over the 12-month study period, two or four measurement points are included across the various outcomes (see figures 1 and 2). At baseline (ie, $\mathrm{t}_{0}$ ), participants will attend a testing 


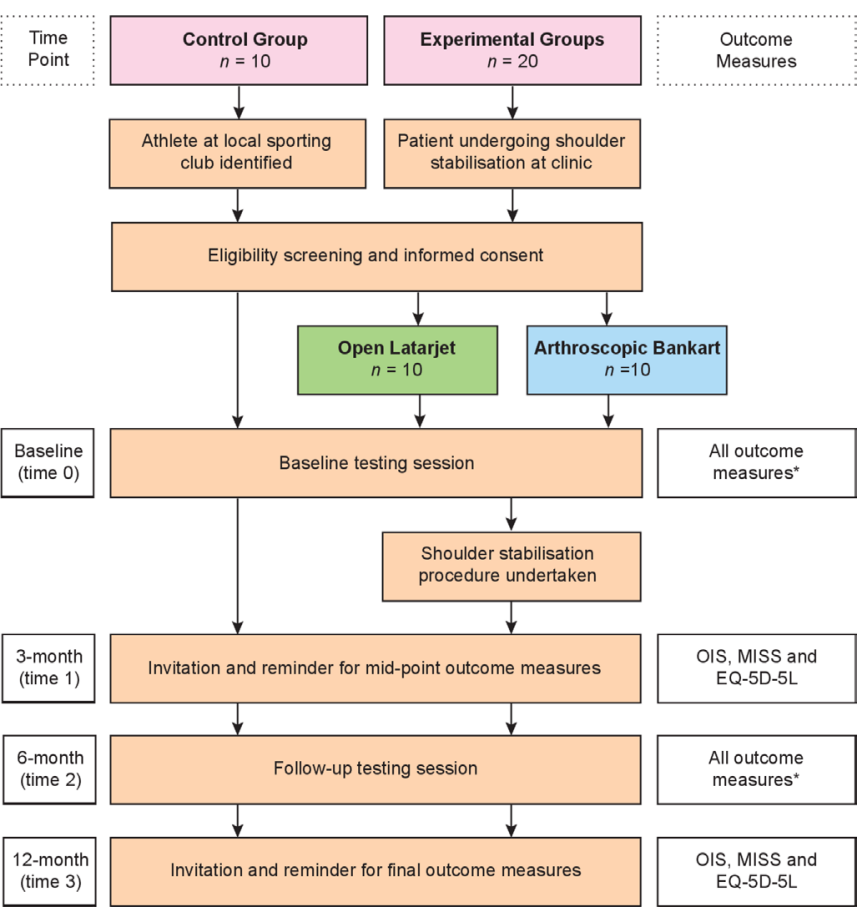

Figure 1 Schematic of participant flow through study protocol. MISS, Melbourne Instability Shoulder Scale; OIS, Oxford Instability Score.

session where all outcome measures will be assessed. At 3 months (ie, $\mathrm{t}_{1}$ ), participants will be invited to complete the questionnaires pertaining to self-reported shoulder function and health status. At 6 months (ie, $\mathrm{t}_{2}$ ), participants will be invited to attend the follow-up testing session where all outcome measures will be measured. At 12 months (ie, $\mathrm{t}_{3}$ ), participants will again be invited to

\begin{tabular}{|c|c|c|c|c|c|c|c|}
\hline \multirow[b]{3}{*}{ TIMEPOINT } & \multicolumn{7}{|c|}{ STUDY PERIOD } \\
\hline & \multirow{2}{*}{$\begin{array}{c}\text { Enrolment } \\
-t_{2} \\
\end{array}$} & \multirow{2}{*}{$\begin{array}{c}\text { Allocation } \\
-t_{1}\end{array}$} & \multicolumn{5}{|c|}{ Post-allocation } \\
\hline & & & to & $\begin{array}{c}\text { Post } \\
t_{0}\end{array}$ & $t_{1}$ & $t_{2}$ & $t_{3}$ \\
\hline \multicolumn{8}{|l|}{ ENROLMENT: } \\
\hline \multirow[t]{2}{*}{ Eligibility screen } & $x$ & & & & & & \\
\hline & $x$ & & & & & & \\
\hline $\begin{array}{r}\text { Informed consent } \\
\text { Surgery }\end{array}$ & & & & $x$ & & & \\
\hline Allocation & & $\mathrm{x}$ & & & & & \\
\hline \multicolumn{8}{|l|}{ INTERVENTIONS: } \\
\hline Arthroscopic Bankart & & & & $x$ & & & \\
\hline \multirow[t]{2}{*}{ Open Latarjet } & & & & $x$ & & & \\
\hline & & & & & & & \\
\hline \multicolumn{8}{|l|}{ ASSESSMENTS: } \\
\hline \multirow{4}{*}{ Self-Reported Shoulder Function } & & & $x$ & & & $x$ & \\
\hline & & & $x$ & & & $x$ & \\
\hline & & & $x$ & & $x$ & $x$ & $\mathrm{X}$ \\
\hline & & & $x$ & & & $x$ & \\
\hline Shoulder Strength & & & $x$ & & & $x$ & \\
\hline
\end{tabular}

Figure 2 Time points of procedures and data collection during study period. complete the questionnaires pertaining to self-reported shoulder function and health status. The study conforms to the Standard Protocol Items: Recommendations for Intervention Trials.

\section{Study population}

Participants in this study will be men and women, between the ages of 18 and 40 years, currently or recently (ie, within the past 12 months) engaged in an overhead contact sport. Overhead contact sports included, but not limited to, in this study will be Australian rules football, rugby league or union, and lacrosse. Those referred to the Geelong Orthopaedic Group or Barwon Health Outpatient clinics who are designated to undergo shoulder stabilisation surgery will be invited to participate and act as the experimental groups. All patients attending the study site for preoperative assessment of shoulder stabilisation surgery will be assessed for eligibility by the surgeon, orthopaedic registrar or research coordinator (see table 1). Athletes with no history of shoulder instability or upper limb surgery will also be recruited through local sporting leagues and act as the control group, and be assessed against the same eligibility criteria. Eligible participants will be invited to participate in the study and informed written consent will be obtained. Participation in the study is voluntary. Recruitment is expected to occur over an 18-month period.

\section{Intervention}

Three surgeons perform surgical shoulder stabilisation procedures at the centre, all of whom will be involved in the study. All surgeons involved in this study are fellowship trained (Royal Australasian College of Surgeons), have greater than 10 years of experience performing upper limb surgical reconstructions, and are currently active members of the Shoulder and Elbow Society of Australia.

Participants in the experimental groups will undergo shoulder stabilisation either via an arthroscopic Bankart repair or an open Latarjet procedure. For the arthroscopic Bankart procedure, individuals will be evaluated under anaesthetic (both a peripheral block and general anaesthesia) to determine the stability pattern of the glenohumeral joint, with clinical findings considered alongside preoperative medical images. ${ }^{30}$ The joint space will be accessed with arthroscopic portals. ${ }^{30}$ The glenohumeral labral tear will be prepared with liberator probes and shaver, and capsulolabral reduction and fixation anteriorly and/or posteriorly will occur with anchors according to the injury pattern. ${ }^{30}$

For the open Latarjet procedure, an oblique $3-5 \mathrm{~cm}$ incision will be made under anaesthesia below the tip of the coracoid process and extending inferiorly down the deltopectoral groove to the superior portion of the axillary fold. ${ }^{24}$ A $22-27 \mathrm{~mm}$ bone graft will be harvested from the tip of the coracoid process, with harvesting and preparation of the graft performed according to standardised procedures and ensuring that the graft is 


\begin{tabular}{|c|c|}
\hline Inclusion criteria & Exclusion criteria \\
\hline $\begin{array}{l}\text { Currently or recently (past } 12 \text { months) engaged in an } \\
\text { overhead contact sport (eg, Australian rules football, rugby } \\
\text { league or union, lacrosse). } \\
\text { Experienced one or more traumatic anterior shoulder } \\
\text { instability injury (ie, subluxation or dislocation) in the past } \\
12 \text { months as determined through clinical evaluation, } \\
\text { resulting in a Bankart repair or Latarjet stabilisation } \\
\text { procedure being deemed as an appropriate treatment } \\
\text { strategy.* } \\
\text { Willing, able and mentally competent to provide informed } \\
\text { consent. }\end{array}$ & $\begin{array}{l}\text { Prior history of shoulder instability injury or self-reported } \\
\text { perceived symptoms of shoulder instability, as deemed by the } \\
\text { Oxford Instability Score questionnaire. } \dagger \\
\text { Prior history of upper limb reconstructive surgery. } \\
\text { Known neurological condition affecting the upper limb. } \\
\text { Presence of adhesive capsulitis of the shoulder-as determined } \\
\text { by self-reported significant loss (ie, }>50 \% \text { ) of shoulder range of } \\
\text { motion. } \neq \\
\text { Presence of a full rotator cuff tear as determined via MRI. } \\
\text { Contraindications for undergoing MRI (eg, metallic foreign } \\
\text { bodies, heart pacemakers). } \\
\text { Foreseeable circumstances that will result in the participant being } \\
\text { unavailable for the 12-month follow-up period. }\end{array}$ \\
\hline
\end{tabular}

${ }^{*}$ Experimental groups only.

†Control group only.

‡Relevant deficits in shoulder range of motion will also be evaluated via standard clinical examination prior to study enrolment.

shaped to be flush with the prepared anterior face of the glenoid. ${ }^{24}$ The anterior portion of the shoulder will be exposed by dividing the subscapularis in line with the muscle fibres, ${ }^{24}$ maintaining a $50-50$ split of the upper and lower portions of the muscle. A T-shaped incision will be made in the labrum at the apex of the bony deformity in preparation to set the coracoid bone graft in place. The coracoid bone graft will be positioned so that it serves as an extension of the inherent articular arc of the glenoid. ${ }^{24}$ Once the desired graft position is identified, fixation of the bone graft to the anterior glenoid will be achieved via two surgical screws under compression. ${ }^{24}$ Anchors will be placed in the native glenoid and the joint capsule repaired. ${ }^{24}$ The subscapularis will be repaired over the coracoid bone graft, with the conjoint tendon exiting anteriorly through the previously split segments of the subscapularis muscle. ${ }^{24}$

Postoperative care of both experimental groups (ie, arthroscopic Bankart and open Latarjet) will follow the clinic's standardised protocols and care pathways. ${ }^{30}$ Participants will use an immobiliser sling for at least 4 weeks post-surgery and then commence a rehabilitation programme to improve active shoulder stability. ${ }^{30}$ Progressive increases to range of movement exercises, strengthening and functional restoration will be incorporated into each participant's individualised programme. ${ }^{30}$ Rehabilitation will be monitored by the clinic for at least 3 months postoperatively. Participants will undergo standard clinical practices for follow-up medical imaging. Participants receiving the open Latarjet treatment will undergo a CT scan at 4 months post-procedure; while participants in both experimental groups will undergo an $\mathrm{X}$-ray 12 months post-procedure. In addition to standard clinical practices, the number of training sessions (both with clinic staff and home-based sessions) the individual completes during the 6-month period following surgery will be monitored via an online survey. Specifically, participants will be prompted to complete an online survey detailing the number and type (ie, range of motion, strength or functional movement) of training sessions they have completed over the past 2 weeks. Return to sport will be assessed after 6 months by the operating surgeon, pending the participant's recovery. ${ }^{30}$ Participants in the control group will be instructed to continue their regular training over the study period.

\section{Assignment of interventions}

The decision as to what stabilisation procedure participants in the experimental group undergoes will be determined by consultation between the individual and the acting surgeon. The primary factor guiding the choice of reconstruction procedure will be the extent of glenoid bone loss and the presence of humeral bone defects, whereby the Latarjet procedure will be more likely in the presence of greater bone loss and/or defects (ie, $>20 \%$ anterior glenoid bone loss).$^{31}$ All participants in the experimental groups will undergo MRI, and where a bone defect is identified be referred for a CT scan. A sagittal view of the glenoid face will be used to fit a circle to the 3-9 o'clock inferior contour of the glenoid. The missing area of this circle (ie, defect) will be determined and presented as a proportion of the total circle area as a means to quantify the bone defect. A secondary factor used in guiding the choice of reconstruction procedure will be the patient's history of shoulder instability, whereby the Latarjet procedure may be more likely in patients with repeat traumatic joint instability. Due to these factors, randomisation of participants as part of this study is not appropriate. Eligible participants will be invited to participate in the study and allocated to the appropriate experimental group based on the procedure they will undergo. Given the different factors driving surgery choice (eg, presence of glenoid or humeral bone loss/defects, prior history of shoulder instability), it is likely that aspects of shoulder pathology and injury history will differ across the experimental groups at baseline in this study. Glenoid bone loss, glenoid and humeral bone defects, injury history and previous surgeries will be 
recorded from participants and any differences in these across groups will be considered in interpreting the final results.

\section{Blinding}

Given the nature of the surgical interventions, the study will be unable to blind participants to the specific stabilisation procedure they receive. Further, the nature of the experimental procedures also means that blinding of assessors will be impossible. During the laboratorybased testing sessions, participants will be required to have markers placed on the skin around the shoulder, leaving this area exposed to the assessor. The presence of surgical scars will reveal whether the participant received the arthroscopic or open procedure. Further, data analysis will involve examining medical imaging data from participants. The analyst will therefore be able to identify the stabilisation procedure performed. Given these factors, no blinding of participants or assessors/analysts will occur.

\section{Data collection and analysis}

Data collection will be performed at four time points $\left(\mathrm{t}_{0}\right.$, $\mathrm{t}_{1}, \mathrm{t}_{2}$ and $\mathrm{t}_{3}$ ). Two of these testing sessions (ie, $\mathrm{t}_{0}$ and $\mathrm{t}_{2}$ ) will include all measures, while $t_{1}$ and $t_{3}$ will only include selfreported shoulder function and health status measures.

\section{Glenohumeral stability and shoulder neuromechanics}

Participants will undergo a laboratory-based biomechanical assessment at $t_{0}$ and $t_{2}$, where they will be evaluated while performing a series of movement tasks. Prior to undertaking the movement tasks, $3814 \mathrm{~mm}$ retro-reflective markers will be affixed to participant's upper limbs and torso (see figure 3). A static calibration trial with the participant standing in the standardised anatomical position will be recorded following marker placement and prior to movement trials. Markers on the medial and lateral epicondyles of the humeri will be removed following the calibration trial. A 10-camera three-dimensional motion capture system (Vicon, Oxford
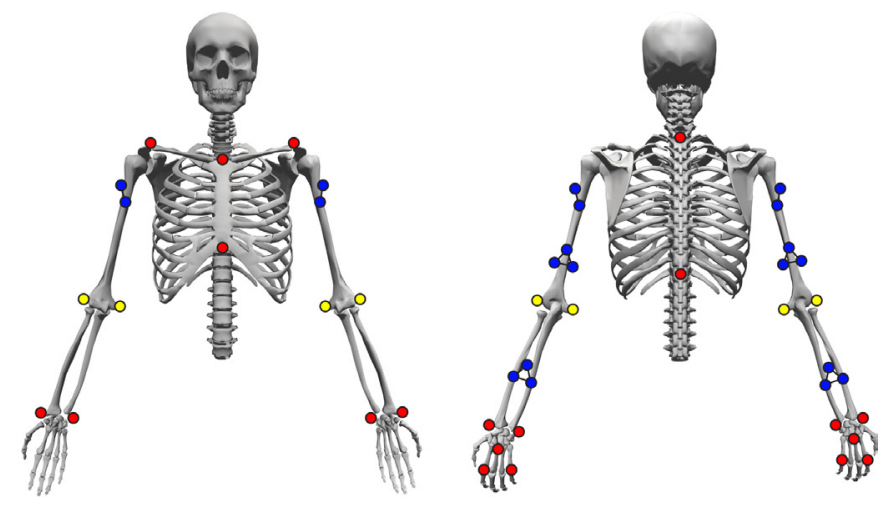

Figure 3 Marker set used to collect biomechanical data. Yellow markers indicate those used in static calibration trials only; blue markers indicate marker clusters remaining for dynamic trials; red markers indicate singular markers remaining for dynamic trials.
Table 2 Functional tasks to be used within laboratorybased testing of shoulder joint neuromechanics (adapted from Vidt et $\mathrm{al}^{32}$ )

\begin{tabular}{|c|c|c|}
\hline Task & Instructions & Load \\
\hline $\begin{array}{l}\text { Forward } \\
\text { reach }\end{array}$ & $\begin{array}{l}\text { Start with hand by side and pick up a } \\
\text { weight on a table at stomach height } \\
\text { at a distance of } 80 \% \text { forearm length; } \\
\text { return hand to starting position with } \\
\text { weight in hand; return weight back to } \\
\text { original position. }\end{array}$ & $1 \mathrm{~kg}^{*}$ \\
\hline $\begin{array}{l}\text { Upward } \\
\text { reach } 90\end{array}$ & $\begin{array}{l}\text { Start with hand by side and pick } \\
\text { up a weight on a shelf at shoulder } \\
\text { height (ie, } 90^{\circ} \text { ); return hand to starting } \\
\text { position with weight in hand; return } \\
\text { weight back to original position. }\end{array}$ & $1 \mathrm{~kg}^{*}$ \\
\hline $\begin{array}{l}\text { Upward } \\
\text { reach } 105\end{array}$ & $\begin{array}{l}\text { Start with hand by side and pick } \\
\text { up a weight on a shelf } 15^{\circ} \text { above } \\
\text { horizontal; return hand to starting } \\
\text { position with weight in hand; return } \\
\text { weight back to original position. }\end{array}$ & $1 \mathrm{~kg}^{*}$ \\
\hline $\begin{array}{l}\text { Sideward } \\
\text { reach }\end{array}$ & $\begin{array}{l}\text { Start with hand by side and pick up a } \\
\text { weight on a shelf at shoulder height } \\
\text { (ie, } 90^{\circ} \text { ) directly to the side; return } \\
\text { hand to starting position with weight } \\
\text { in hand; return weight back to original } \\
\text { position. }\end{array}$ & $1 \mathrm{~kg}^{*}$ \\
\hline
\end{tabular}

Axilla wash Start with hand by side; reach across No the torso to touch the lateral aspect of load the opposite shoulder; return hand to starting position.

Rear reach Start with hand by side; reach behind No the torso to touch the centre of the load small of the back with palm side of fingers/hand; return hand to starting position.

\begin{tabular}{|c|c|c|}
\hline Hair comb & $\begin{array}{l}\text { Start with hand by side; reach to the } \\
\text { forehead; comb the centre of the hair } \\
\text { from front to back once; return hand } \\
\text { to starting position. }\end{array}$ & $\begin{array}{l}\text { No } \\
\text { load } †\end{array}$ \\
\hline
\end{tabular}

${ }^{*} \mathrm{~A} 1 \mathrm{~kg}$ dumbbell to be used to mimic a typical kitchen object (eg, a plate).

†Pencil used to mimic holding a comb.

Metrics Limited, Oxford, UK), sampling at $250 \mathrm{~Hz}$, will be used to track the position of the markers as participants perform each task. Recorded marker trajectories will be smoothed using a low-pass fourth-order Butterworth filter, with the cut-off frequency determined via residual analysis of the marker trajectory data.

Participants will complete seven functional tasks representative of activities of daily living involving the shoulder (see table 2). ${ }^{32}$ These tasks will be performed with the surgical and 'test' limb of the experimental and control groups, respectively. The test limb for control participants will be randomly allocated as their dominant or non-dominant arm (as determined by the limb they write with) - with a 50/50 balance achieved for dominant and non-dominant test limbs within the group. Participants 

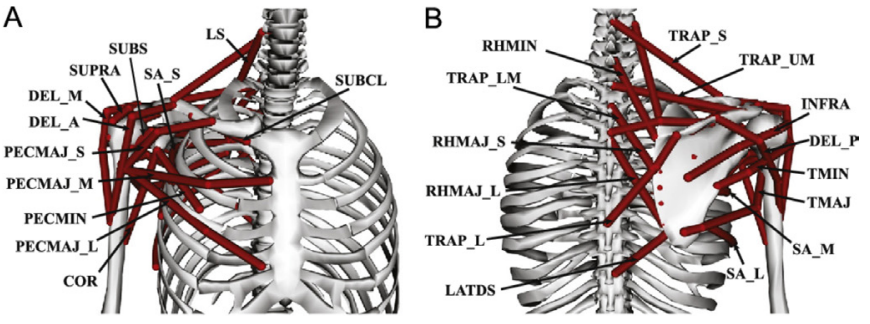

Figure 4 Visual representation of muscles included in the upper limb musculoskeletal model. COR, coracoid process; DEL_A, anterior deltoid; DEL_M, middle deltoid; DEL_P, posterior deltoid; INFRA, infraspinatus; LATDS, latissimus dorsi; LS, levator scapulae; PECMAJ_L, lower pectoralis major; PECMAJ_M, middle pectoralis major; PECMAJ_S, superior pectoralis major; PECMIN, pectoralis minor; RHMAJ_L, lower rhomboid major; RHMAJ_S, superior rhomboid major; RHMIN, rhomboid minor; SA_L, lower serratus anterior; SA_M, middle serratus anterior; SA_S, superior serratus anterior; SUBCL, subclavius; SUBS, subscapularis; SUPRA, supraspinatus; TMAJ, teres major; TMIN, teres minor; TRAP_L, lower trapezius; TRAP_LM, lower middle trapezius; TRAP_S, superior trapezius; TRAP UM, upper middle trapezius.

will be instructed on functional task start and end positions, but will be able to freely choose their joint postures and speed of movement. ${ }^{32} 33$

An activity that simulates an overhead sporting task that requires participants to jump and reach to an overhead target will also be performed. The overhead sporting task will consist of a 6-metre run-up, followed by a single-leg jump to reach a ball placed at the participant's maximal vertical jump height (determined via standardised methods prior to performing this task). The ball will be attached to a specially made ball rig via Velcro, ensuring that the participant can reach and remove the ball during task performance.

Participants will also perform an extreme range of motion trial with their surgical or test limb. This trial will involve the participant keeping the elbow and wrist in a neutral position (ie, arm straight, $0^{\circ}$ of elbow flexion, and wrist flexion and deviation) and moving the shoulder to its extreme ranges of motion (within participants' comfortable limits) in all directions. This motion will be used to create a three-dimensional range of motion 'globe' to which the other movement trials can be mapped on. ${ }^{34}$

All functional, sporting and range of motion tasks will be repeated (with 30 and 60 s rest given between task trials, respectively) until three successful trials are recorded. A successful trial for the functional tasks will be characterised by the participant performing the task with the desired start and end positions, and meeting the task instructions. Successful trials of the sporting task will be characterised by the participant jumping off a single leg, and reaching and removing the ball from the rig in a smooth motion. The order of tasks will be randomised across participants.

Participant-specific musculoskeletal models of the upper limb will be generated and used in the analysis of laboratory-based biomechanical data from $t_{0}$ and $t_{2}$. A generic seven segment (torso, clavicle, scapula, humerus, ulna, radius and hand) musculoskeletal model of the torso and upper limb will serve as the basis for the participant-specific models developed in this study. The kinematic foundation for the model ${ }^{3536}$ will describe the kinematics of the shoulder girdle (ie, shoulder elevation, elevation plane and shoulder rotation), elbow (ie, elbow flexion) and forearm (ie, pronation/supination) as recommended by the International Society of Biomechanics. ${ }^{37}$ Overall motion of the shoulder girdle (including the clavicle and scapula will be determined by the regression equations described by de Groot and Brand, ${ }^{38}$ and driven by the shoulder elevation angle. ${ }^{35} 36$ The wrist and finger joints will be locked to minimise the df in the model, which will reduce simulation complexity and computation time. The model will be actuated by 26 Hill-type muscle-tendon units representing the main axioscapular, axiohumeral and scapula-humeral muscle groups (see figure 4$).{ }^{39}$ Idealised torque actuators will be used to drive elbow and forearm motions. Model parameters (ie, segment inertial properties, muscle strength estimates, muscle attachment points and paths) will be estimated from MRI data using established procedures. $^{4041}$ Participants in the experimental groups will undergo MRI scans prior to both laboratory-based testing sessions, with separate models created for each testing session. This approach will ensure the models take into account any changes in shoulder anatomy introduced by the stabilisation procedure. Participants in the control group will undergo one scan at the commencement of the study, with this used to create the model for both testing sessions (as no changes in shoulder anatomy are expected in this group).

Biomechanical simulations of the movement tasks will be performed in OpenSim ${ }^{42}$ (V.4.0) using the MATLAB V.R2019b (MathWorks, Natick, Massachusetts, USA) API and participant-specific models. Torso and upper limb joint angles and moments will be calculated using the inverse kinematics and dynamics OpenSim functions, respectively. Activations and forces of the shoulder muscles during the movement tasks will be estimated using a static optimisation approach. The static optimisation process will aim to minimise the sum of squared muscle and torque actuator activations required to generate the movement, while also constraining the glenohumeral joint reaction force direction. ${ }^{39}$ This additional constraint aims to simulate rotator cuff muscle co-contraction, and ensures that the calculated muscle forces produce sufficient glenohumeral joint compression and stabilisation. ${ }^{39}$ Following the optimisation, the joint reaction force function in OpenSim will be used to calculate the glenohumeral joint reaction force applied to the scapula body in the humeral joint coordinate system (x-axis=anterior-posterior; $\quad \mathrm{y}$-axis=superior-inferior; $\mathrm{z}$-axis=medial-lateral). Joint kinematics and moments, muscle activations and forces, and joint reaction forces across each movement will be normalised to a time-scale 
of $0 \%-100 \%$ of the movement. Joint kinematics of each task will also be used to plot the trajectory of the upper limb (humerus segment) on the globe generated from the extreme range of motion task. ${ }^{34}$ The area of the globe covered by each movement task will be divided by the total area of the globe, resulting in a percentage representing the amount of shoulder mobility used in the task. $^{34}$

Glenohumeral stability across the movement tasks examined will be estimated using the direction of the glenohumeral joint reaction force vector in the scapula plane. ${ }^{43-45}$ The stability of the joint will be defined as a function of the angle of the resultant joint reaction force vector in the glenoid relative to the maximum angle required to reach the outer edge of the glenoid. ${ }^{434}$ The stability value will be calculated at each step across the time-normalised movement tasks. A stability value of zero will occur when the resultant force is directed to the centre of the glenoid, with the stability value increasing towards one (which is the theoretical maximum value that can be reached before dislocation of the joint) as the vector moves towards the outside of the glenoid. ${ }^{434}$ Medical imaging data (ie, MRI) will be used to fit a twodimensional ellipse to a lateral view of the glenoid fossa surface for calculations of glenohumeral stability.

\section{Shoulder range of motion and strength}

During laboratory-based testing (ie, at $\mathrm{t}_{0}$ and $\mathrm{t}_{2}$ ), participants will also undergo assessment of their shoulder range of motion and strength. Shoulder range of motion will be evaluated in the directions of abduction, flexion, extension (hyperextension), internal and external rotation at $45^{\circ}$ of abduction, and external rotation at $90^{\circ}$ of abduction. The order for the range of motion tests will be randomised across participants. Participants will be positioned in a dynamometer (Biodex, Shirley, New York, USA) and be instructed to move through their maximum range of motion (ie, to the point where they can no longer move any further) in the specified direction. All directions will be performed with the participant in a seated position (ie, hips and knees at $90^{\circ}$ of flexion) with their back firmly pressed against the dynamometer chair. Straps affixed to the dynamometer chair will be placed across the participant's chest to avoid any movement of the body during the motion. The participant will be positioned so that the axis of rotation of the dynamometer arm is in line with the shoulder joint centre across all tests. The shoulder abduction, flexion and extension (hyperextension) tests will begin with the participant's arm placed by their side, with the palm in a neutral position (ie, palm facing the thigh), and initiated by the participant moving their arm in the respective directions until the maximum range of motion is reached. Shoulder internal and external rotation range of motion will be tested with the arm abducted to a position of $45^{\circ}$ and the elbow flexed at $90^{\circ}$, while external rotation range of motion will be further tested with the arm abducted to a position of $90^{\circ}$ and the elbow flexed at $90^{\circ}$. Participants will internally or externally rotate at the shoulder from the starting positions to their maximum range of motion. The displacement achieved from the starting position will be recorded as the participant's range of motion (in degrees) across all tests. Range of motion tests will be ceased if participants report any feelings of shoulder instability. Following the active range of motion tests driven by participants, a passive test will be conducted by programming the dynamometer to move the participant through their range of motion with no active muscle contraction. The dynamometer will calculate the passive torque (in $\mathrm{Nm}$ ) generated through the participant's range of motion in the respective positions and directions. The passive torque generated will be timenormalised to a scale of $0 \%-100 \%$ of the participant's range of motion.

Strength testing will also be conducted using the dynamometer. Isometric strength in the directions of abduction, flexion and internal/external rotation will be evaluated in a range of positions (see table 3 ). The dynamometer will be locked in the respective positions for each test. The participant will be instructed to perform a maximal effort concentric contraction by steadily building to maximum effort over $3 \mathrm{~s}$; holding the maximum effort for $1 \mathrm{~s}$ and steadily returning to no effort over 3 s. Participants will perform three maximal efforts across each position, with $1 \mathrm{~min}$ of rest allocated between each effort to minimise the effect of fatigue. The dynamometer will record the torque (in $\mathrm{Nm}$ ) produced across each contraction, with the maximum torque achieved across any repetition for the respective movement directions

Table 3 Positions and limbs used for each isometric strength test

\begin{tabular}{|c|c|c|c|}
\hline Direction & Position(s)* & $\begin{array}{l}\text { 'Test'/surgical } \\
\text { limb }\end{array}$ & Opposite limb \\
\hline \multirow[t]{2}{*}{ Abduction } & Neutral ( $0^{\circ}$ of abduction) & $\mathrm{x}$ & \\
\hline & Horizontal ( $90^{\circ}$ of abduction) & $\mathrm{X}$ & $\mathrm{x}$ \\
\hline Flexion & Neutral ( $0^{\circ}$ of flexion) & $\mathrm{X}$ & $\mathrm{X}$ \\
\hline \multirow{2}{*}{$\begin{array}{l}\text { Internal/external } \\
\text { rotation }\end{array}$} & Horizontal with elbow flexed ( $45^{\circ}$ of abduction; $0^{\circ}$ of external rotation; $90^{\circ}$ of elbow flexion) & $\mathrm{x}$ & \\
\hline & $\begin{array}{l}\text { Horizontal and rotated with elbow flexed }\left(90^{\circ} \text { of abduction and external rotationt; } 90^{\circ} \text { of }\right. \\
\text { elbow flexion) }\end{array}$ & $x$ & $\mathrm{x}$ \\
\hline
\end{tabular}

${ }^{*}$ A straight arm (ie, $0^{\circ}$ of elbow flexion) will be used across all tests, unless noted otherwise.

†The maximal external rotation angle achievable if less than $90^{\circ}$. 
and positions used as the indicator of isometric shoulder strength. All isometric strength tests will be performed on the test limb, with three tests (see table 3) repeated on the opposite side. This will serve as a within-individual comparison of side-to-side strength imbalance between limbs.

Eccentric isokinetic strength will be assessed in the directions of flexion and external/internal rotation, in postures associated with anterior instability injuries in contact sport athletes. These tests are designed to assess eccentric strength in shoulder positions associated with anterior instability injuries in contact sport athletes, namely: (1) external rotation in an abducted position ${ }^{46} 47$ and (2) flexion with an outstretched arm. ${ }^{47} 48$ The flexion test will involve the participant starting with the arm in a horizontal position in front of the body (ie, $0^{\circ}$ of flexion). The test will then involve the participant resisting the motion of the dynamometer with a maximal effort contraction as the shoulder is slowly moved into flexion and relax as the dynamometer moves them back to the starting position. The external/internal rotation test will involve the participant starting with the elbow flexed at $90^{\circ}, 90^{\circ}$ of shoulder abduction and $0^{\circ}$ of external rotation. The test will then involve the participant resisting the motion of the dynamometer with a maximal effort contraction as the shoulder is slowly moved into external rotation; and then also resist the motion of the dynamometer with a maximal contraction as it moves them back through internal rotation to the starting position. Isokinetic strength testing will be tested at $60^{\circ} \% \mathrm{~s}$, and as such the dynamometer arm will move at this speed throughout the respective motions. Each isokinetic test will involve the dynamometer arm moving through the dictated range of motion and back for three repetitions, with the dynamometer recording the torque produced (in $\mathrm{Nm}$ ) throughout the motion. To ensure participants are not placed in a high-risk position for instability, participants will only be tested to positions of $75 \%$ of their maximum flexion and external rotation angles. Data from each trials repetitions will be collated and averaged to obtain mean eccentric torque across the different movement directions. The maximum torque achieved across all trials for the different movement directions will be identified and recorded as the peak torque. The order for the different isometric and eccentric strength tests will be randomised across participants, and any test will cease if participants report feelings of instability.

\section{Self-reported shoulder function and health status}

Self-report measures of shoulder function and health status will be recorded at all time points (ie, $\mathrm{t}_{0}, \mathrm{t}_{1}, \mathrm{t}_{2}$ and $t_{3}$ ) through an online survey (REDCap, Vanderbilt University, Nashville, Tennessee, USA). Self-reported shoulder function will be assessed using the Oxford Instability Score (OIS) ${ }^{49}$ and Melbourne Instability Shoulder Scale (MISS) ${ }^{50}$; while self-reported general health status will be assessed using the EQ-5D-5L. ${ }^{51}$ The three survey instruments will be included within one online survey that participants will complete at the appropriate time points. The survey will be sent to participants in the experimental groups 2 weeks prior to their scheduled shoulder stabilisation procedure and at the follow-up time points. The same schedule will be followed for control participants, with the exception of the first survey being sent to the participant upon study enrolment. The total and section scores for each of the different survey instruments (ie, the OIS, MISS and EQ-5D-5L) will be calculated according to their established methods ${ }^{4-51}$ for each participant at each time point.

\section{Statistical analysis}

Sample size calculations are based on the primary outcome of glenohumeral stability. To our knowledge, no specific data pertaining to the metric of glenohumeral stability are available for individuals following shoulder stabilisation surgery; nor is there any indication in the literature as to what is deemed to be a clinically important difference for this metric. As such, sample size calculations are based on data presented in Marchi et $a l,{ }^{45}$ which examined glenohumeral stability in individuals with a history of shoulder joint dislocation during hand-positioning tasks. Mean and SD in this existing study for glenohumeral stability was $0.655 \pm 0.023 .{ }^{45} \mathrm{We}$ have assumed a conservative difference of $5 \%$ (ie, 0.033) to represent a clinically important difference. Based on this difference, a sample size of 27 participants (ie, nine per group) is calculated with a power $(1-\beta)$ of $80 \%$ and a type I error $(\alpha)$ of $5 \%$. This study will therefore include a total of 30 participants (10 per group) to meet the computed sample size while allowing for $10 \%$ dropout. To compensate for any potential insufficiencies in our a priori sample size calculation (due to lack of comparable studies), we will perform post-hoc power calculations to evaluate any beta error. Additionally, all differences will be presented and interpreted with $95 \%$ CIs and effect size estimates.

Data will be compared across groups and time points using a two-way (group xtime) analysis of variance (ANOVA) with repeated measures on one factor (ie, time) design or the non-parametric equivalent should data not be normally distributed (as assessed by a Shapiro-Wilk test). The discrete outcome measures of glenohumeral joint stability, shoulder strength, shoulder range of motion and self-report measure scores will be submitted to a traditional ANOVA using the aforementioned design. The continuous outcome measures of joint kinematics and moments, muscle activations and forces, and joint reaction forces will be submitted to an equivalent ANOVA design using vector-field onedimensional statistical parametric mapping. Relevant post-hoc comparisons (with Bonferroni corrections applied) will be made between groups and time points where ANOVA detects a statistically significant difference ( $\mathrm{p}<0.05$ adjusted for the multiple comparisons made). The $95 \%$ CIs and estimates of effect sizes (ie, Cohen's d) 
will also be calculated for differences between and within groups across the time points.

\section{Patient and public involvement}

Patients and/or the public were not involved in the design, or conduct, or reporting, or dissemination plans of this research.

\section{Twitter Aaron Fox @aaron_s_fox}

Contributors AF and RSP conceived the study. AF oversaw manuscript writing and submission. AF, JB, SDG and RSP contributed to the design of the study protocol, assisting with drafting the manuscript, and have read and approved the final version of the manuscript.

Funding This work is partially supported by a Deakin University Postdoctoral Research Fellowship grant (held by AF). Funding will be used to pay for costs associated with data collection and analysis.

Competing interests RSP receives institutional educational support from De Puy Synthes, New Brunswick, New Jersey, USA. AF, JB and SDG report no competing interests.

\section{Patient consent for publication Not required.}

Ethics approval This study will be conducted in accordance with the Declaration of Helsinki and the Australian National Health and Medical Research Council's National Statement on Ethical Conduct in Human Research. The study protocol has been approved by the Barwon Health (project number 19-53) and Deakin University Human Research Ethics (project number 2019-419) committees. All collected data will be stored electronically using a re-identifiable coding system for participants. This will ensure that data are stored and used in the strictest confidence and will not reveal participant identity. Electronic data will be stored on secure servers only accessible to the project lead and associate investigators. The results will be submitted for publication in peer-reviewed journals and for presentation at relevant scientific conferences. Where appropriate, we also aim to disseminate the results through relevant media outlets (eg, specialised magazines, websites). No data or participant information will be identifiable in the disseminated results. Any specific individual data reported will be non-identifiable.

Provenance and peer review Not commissioned; externally peer reviewed.

Data availability statement No data are available. This paper represents a study protocol, hence no data have been collected.

Open access This is an open access article distributed in accordance with the Creative Commons Attribution Non Commercial (CC BY-NC 4.0) license, which permits others to distribute, remix, adapt, build upon this work non-commercially, and license their derivative works on different terms, provided the original work is properly cited, appropriate credit is given, any changes made indicated, and the use is non-commercial. See: http://creativecommons.org/licenses/by-nc/4.0/.

\section{ORCID iD}

Aaron Fox http://orcid.org/0000-0002-5639-6388

\section{REFERENCES}

1 Leclere LE, Asnis PD, Griffith MH, et al. Shoulder instability in professional football players. Sports Health 2013;5:455-7.

2 Orchard JW, Seward H, Orchard JJ. Results of 2 decades of injury surveillance and public release of data in the Australian football League. Am J Sports Med 2013;41:734-41.

3 Bohu Y, Klouche S, Lefevre N, et al. The epidemiology of 1345 shoulder dislocations and subluxations in French rugby union players: a five-season prospective study from 2008 to 2013. Br J Sports Med 2015;49:1535-40.

4 Thangarajah T, Lambert S. Management of the unstable shoulder. $\mathrm{Br}$ J Sports Med 2016;50:440-7.

5 Owens BD, Nelson BJ, Duffey ML, et al. Pathoanatomy of first-time, traumatic, anterior glenohumeral subluxation events. J Bone Joint Surg Am 2010;92:1605-11.

6 Itoi E, Yamamoto N, Kurokawa D, et al. Bone loss in anterior instability. Curr Rev Musculoskelet Med 2013;6:88-94.

7 Milano G, Grasso A, Russo A, et al. Analysis of risk factors for glenoid bone defect in anterior shoulder instability. Am J Sports Med 2011;39:1870-6.

8 Calandra JJ, Baker CL, Uribe J. The incidence of Hill-Sachs lesions in initial anterior shoulder dislocations. Arthroscopy 1989;5:254-7.
9 Zacchilli MA, Owens BD. Epidemiology of shoulder dislocations presenting to emergency departments in the United States. J Bone Joint Surg Am 2010;92:542-9.

10 Robinson CM, Howes J, Murdoch $\mathrm{H}$, et al. Functional outcome and risk of recurrent instability after primary traumatic anterior shoulder dislocation in young patients. J Bone Joint Surg Am 2006;88:2326-36.

11 Mohtadi NGH, Chan DS, Hollinshead RM, et al. A randomized clinical trial comparing open and arthroscopic stabilization for recurrent traumatic anterior shoulder instability. J Bone Joint Surg 2014;96:353-60.

12 Rolfes K. Arthroscopic treatment of shoulder instability: a systematic review of capsular plication versus thermal capsulorrhaphy. J Athl Train 2015;50:105-9.

13 Berendes TD, Pilot P, Nagels J, et al. Survey on the management of acute first-time anterior shoulder dislocation amongst Dutch public hospitals. Arch Orthop Trauma Surg 2015;135:447-54.

14 Millett PJ, Clavert P, Warner JJP. Open operative treatment for anterior shoulder instability: when and why? J Bone Joint Surg Am 2005;87:419-32.

15 Latarjet M. A propos du traitement des luxations récidivante de l'épaule. Lyon Chir 1954;49:994-1003.

16 Bonazza NA, Liu G, Leslie DL, et al. Trends in surgical management of shoulder instability. Orthop J Sport Med 2017;5:232596711771247.

17 Page R, Fraser-Moodie J, Mow T. The ‘Down Under' Lesion. Inferior labral pathology in shoulder instability: Epidemiology and minimum 2 year follow-up. Jeju, Korea: 13th International Congress of Shoulder and Elbow Surgery, 2016.

18 Bonacci J, Manson B, Bowe SJ, et al. Operative shoulder instability injury management in Australian football League players: a case series. J Sci Med Sport 2018;21:760-4.

19 Bessière $\mathrm{C}$, Trojani $\mathrm{C}$, Carles $\mathrm{M}$, et al. The open latarjet procedure is more reliable in terms of shoulder stability than arthroscopic bankart repair. Clin Orthop Relat Res 2014;472:2345-51.

20 Neyton L, Young A, Dawidziak B, et al. Surgical treatment of anterior instability in rugby union players: clinical and radiographic results of the Latarjet-Patte procedure with minimum 5-year follow-up. $J$ Shoulder Elbow Surg 2012;21:1721-7.

21 Schmiddem U, Watson A, Perriman D. Arthroscopic repair of HAGL lesions yields good clinical results, but may not allow return to former level of sport. Knee Surgery, Sport Traumatol Arthrosc 2020.

22 Williams HLM, Evans JP, Furness ND. It's Not All About Redislocation: A Systematic Review of Complications After Anterior Shoulder Stabilization Surgery. Am J Sports Med 2018;036354651881071.

23 Brophy RH, Marx RG. Osteoarthritis following shoulder instability. Clin Sports Med 2005;24:47-56.

24 Bhatia S, Frank RM, Ghodadra NS, et al. The outcomes and surgical techniques of the latarjet procedure. Arthroscopy 2014;30:227-35.

25 Ghodadra N, Gupta A, Romeo AA, et al. Normalization of glenohumeral articular contact pressures after Latarjet or iliac crest bone-grafting. J Bone Joint Surg Am 2010;92:1478-89.

26 Schmiddem U, Hawi N, Liodakis E, et al. Monocortical fixation of the coracoid in the Latarjet procedure is significantly weaker than bicortical fixation. Knee Surg Sports Traumatol Arthrosc 2019;27:239-44.

27 An VVG, Sivakumar BS, Phan K, et al. A systematic review and meta-analysis of clinical and patient-reported outcomes following two procedures for recurrent traumatic anterior instability of the shoulder: Latarjet procedure vs. Bankart repair. J Shoulder Elbow Surg 2016;25:853-63.

28 Park I, Lee J-H, Hyun H-S, et al. Minimal clinically important differences in Rowe and Western Ontario shoulder instability index scores after arthroscopic repair of anterior shoulder instability. $J$ Shoulder Elbow Surg 2018;27:579-84.

29 Cools AM, Borms D, Castelein B, et al. Evidence-Based rehabilitation of athletes with glenohumeral instability. Knee Surg Sports Traumatol Arthrosc 2016;24:382-9.

30 Page RS, McGee SL, Eng K, et al. Adhesive capsulitis of the shoulder: protocol for the adhesive capsulitis biomarker (AdCaB) study. BMC Musculoskelet Disord 2019;20:145.

31 Rabinowitz J, Friedman R, Eichinger JK. Management of Glenoid bone loss with anterior shoulder instability: indications and outcomes. Curr Rev Musculoskelet Med 2017;10:452-62.

32 Vidt ME, Santago AC, Marsh AP, et al. The effects of a rotator cuff tear on activities of daily living in older adults: a kinematic analysis. $J$ Biomech 2016;49:611-7.

33 Vidt ME, Santago AC, Marsh AP, et al. Modeling a rotator cuff tear: individualized shoulder muscle forces influence glenohumeral joint contact force predictions. Clin Biomech 2018;60:20-9. 
34 BBS Y, Da CJS, AKS Y. Global Arc of motion: an alternative way of describing shoulder kinematics, a study on activities of daily living. Dublin, Ireland: Proceedings of the 8th World Congress of Biomechanics, 2018.

35 Holzbaur KRS, Murray WM, Delp SL. A model of the upper extremity for simulating musculoskeletal surgery and analyzing neuromuscular control. Ann Biomed Eng 2005;33:829-40.

36 Saul KR, Hu X, Goehler CM, et al. Benchmarking of dynamic simulation predictions in two software platforms using an upper limb musculoskeletal model. Comput Methods Biomech Biomed Engin 2015;18:1445-58.

37 Wu G, van der Helm FCT, Veeger HEJD, et al. ISB recommendation on definitions of joint coordinate systems of various joints for the reporting of human joint motion--Part II: shoulder, elbow, wrist and hand. J Biomech 2005;38:981-92.

38 de Groot JH, Brand R. A three-dimensional regression model of the shoulder rhythm. Clin Biomech 2001;16:735-43.

39 Wu W, Lee PVS, Bryant AL, et al. Subject-Specific musculoskeletal modeling in the evaluation of shoulder muscle and joint function. $J$ Biomech 2016;49:3626-34.

40 Valente G, Crimi G, Vanella N, et al. nmsBuilder: freeware to create subject-specific musculoskeletal models for OpenSim. Comput Methods Programs Biomed 2017;152:85-92.

41 Modenese L, Montefiori E, Wang A, et al. Investigation of the dependence of joint contact forces on musculotendon parameters using a codified workflow for image-based modelling. J Biomech 2018;73:108-18.
42 Delp SL, Anderson FC, Arnold AS, et al. OpenSim: open-source software to create and analyze dynamic simulations of movement. IEEE Trans Biomed Eng 2007;54:1940-50.

43 Chadwick EK, Blana D, van den Bogert AJT, et al. A real-time, 3-D musculoskeletal model for dynamic simulation of arm movements. IEEE Trans Biomed Eng 2009;56:941-8.

44 Chadwick EK, Blana D, Kirsch RF, et al. Real-Time simulation of three-dimensional shoulder girdle and arm dynamics. IEEE Trans Biomed Eng 2014;61:1947-56.

45 Marchi J, Blana D, Chadwick EK. Glenohumeral stability during a hand-positioning task in previously injured shoulders. Med Biol Eng Comput 2014;52:251-6.

46 Cutts S, Prempeh M, Drew S. Anterior shoulder dislocation. Ann R Coll Surg Engl 2009;91:2-7.

47 Mattern O, Funk L, Walton MJ. Anterior shoulder instability in collision and contact athletes. Arthroscopy 2018;5:99-106.

48 Crichton J, Jones DR, Funk L. Mechanisms of traumatic shoulder injury in elite rugby players. Br J Sports Med 2012;46:538-42.

49 Dawson J, Fitzpatrick R, Carr A. The assessment of shoulder instability. the development and validation of a questionnaire. J Bone Joint Surg Br 1999;81:420-6.

50 Watson L, Story I, Dalziel R, et al. A new clinical outcome measure of glenohumeral joint instability: the miss questionnaire. J Shoulder Elbow Surg 2005;14:22-30.

51 EuroQol Research Foundation. ED-5D-5L user guide: basic information on how to use the EQ-5D-5L instrument, 2015. 